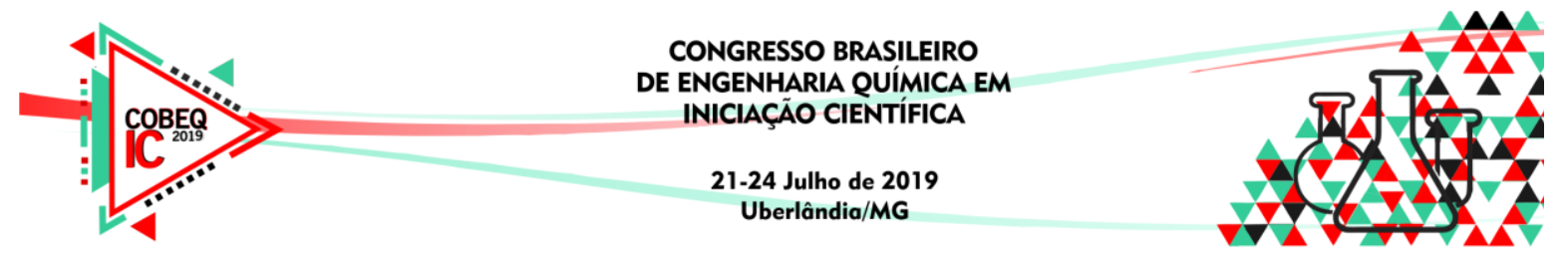

\title{
INFLUÊNCIA DOS MÉTODOS DE PARTICIONAMENTO NO CUSTO COMPUTACIONAL EM SIMULAÇÕES VIA CFD PARA HIDROCICLONES
}

\author{
COSTA, F.O. e PETRI JUNIOR, I. \\ ${ }^{1}$ Universidade Federal de Lavras - Departamento de Engenharia \\ E-mail para contato: felipe.costa@estudante.ufla.br
}

\begin{abstract}
RESUMO - Em simulação utilizando computadores multiparticionados, a forma com que a malha é particionada afeta diferentemente no tempo médio por iteração, consequentemente, no custo computacional. Este trabalho propõe analisar o tempo médio por iteração dos 23 métodos de partição disponíveis para malhas tridimensionais do software FLUENT $^{\circledR} 19.2$ versão acadêmica, vale ressaltar que algumas partições fazem exatamente a mesma divisão da malha. Foram feitas coletas de resultados em triplicatas, para assim, efetuar o cálculo do desvio padrão, que representa as oscilações da capacidade de processamento do computador utilizado e foi utilizado 100 iteração para coletar o tempo médio por iteração. No geral, os melhores métodos de partição foram aqueles em que a divisão da malha foi feita perpendicularmente ao eixo no qual o equipamento está disposto, e quando a partição é feita perpendicularmente aos demais eixos os tempos médios por iteração são mais elevados. Sendo assim, a escolha do método de partição adequado pode economizar um alto custo computacional, para o hidrociclone utilizado, com um computador com 8 núcleo pode-se economizar aproximadamente 24,56 horas, representado quase $20 \%$ do tempo total.
\end{abstract}

\section{INTRODUÇÃO}

Em simulações utilizando computadores multiparticionados, a maneira na qual a malha será dividido influenciará no tempo gasto na simulação. Portanto, esse parâmetro possui grande influência no custo computacional envolvido no processo. O software FLUENT $^{\circledR}$ possui vinte e três métodos de particionamento para malhas tridimensionais, sendo eles Cartesian Axes, Cartesian Strip, Cartesian X-Coordinate, Cartesian Y-Coordinate, Cartesian Z-Coordinate, Cartesian $R$ Axes, Cartesian RX-Coordinate, Cartesian RY-Coordinate, Cartesian RZCoordinate, Cylindrical Axes, Cylindrical R-Coordinate, Cylindrical Theta-Coordinate, Cylindrical Z-Coordinate, Metis, Principal Axes, Principal Strip, Principal X-Coordinate, Principal Y-Coordinate, Principal Z-Coordinate, Spherical Axes, Spherical Rho-Coordinate, Spherical Theta-Coordinate e Spherical Phi-Coordinate (ANSYS, 2018).

Todos os métodos de partição apresentam algumas características principais em comum, sendo elas, manter aproximadamente o mesmo número de células em cada partição, minimizar o número de interfaces de partições, isto é, diminuir a área superficial limite da partição e minimizar o número de partições vizinhas. Cada um dos métodos de partição se adapta a geometria do equipamento e a partir desses princípios gerais, é formada a partição, sendo que, o número de partições está relacionado ao número de núcleos do computador multiparticionado, 


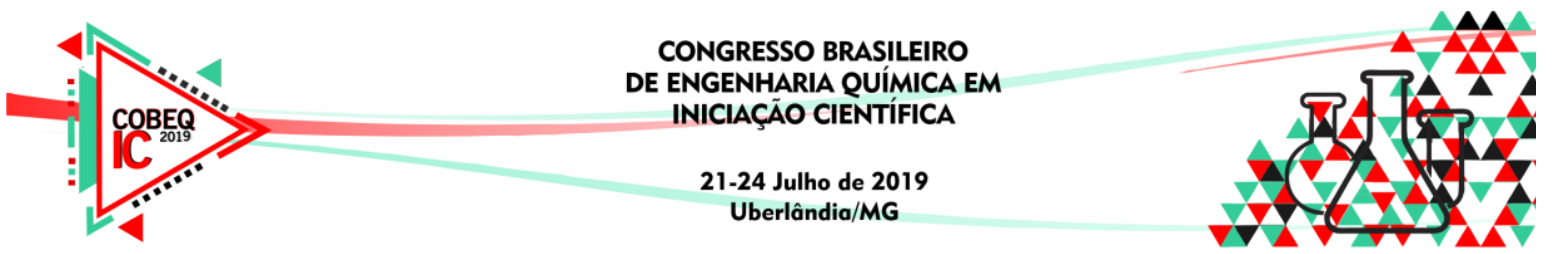

entretanto a forma na qual malha será dívida depende da geometria do equipamento e do método aplicado (ANSYS, 2018).

O principal objetivo, de cada uma das partições possuírem aproximadamente o mesmo número de células, é de não sobrecarregar nenhum dos núcleos do computador, para que cada um dos processadores possuam uma carga nivelada para se comunicar entre si com mesma velocidade. Tendo em vista que a comunicação entre as partições é um processo lento, e por isso também as partições são criadas com a menor área superficial possível, para minimizar esse processo de transmissão de informação entre as partições. Minimizar o número de vizinhos de partição reduz as chances de contenção de rede e roteamento, acelerando assim o processo de simulação (ANSYS, 2018).

O software FLUENT $^{\circledR}$ utiliza o algoritmo Metis ou a bissecção para a criação das partições, a principal vantagem desses métodos é que ele não possui um limite quanto ao número de partições criadas, e por isso, o número de partições criadas é o número de núcleo do computador utilizado. O princípio de funcionamento do método da bissecção, consiste na divisão puramente geométrica do domínio principal em subdomínios, já a divisão do domínio principal através do algoritmo Metis é feita por técnicas de particionamento gráficas, sendo na maioria dos casos mais eficiente (ANSYS, 2018).

Sendo assim, o objetivo deste trabalho é estudar os vinte e três métodos de particionamento (para geometrias tridimensionais) disponíveis pelo software FLUENT $^{\circledR}$ do pacote computacional da ANSYS, a fim de minimizar o tempo de simulações em paralelo, para computadores multiparticionados, vale ressaltar que alguns métodos fazem a mesma partição da malha, sendo portando repetidos.

\section{METODOLOGIA}

Os métodos de particionamento influenciam diretamente no tempo de simulação e consequentemente no custo computacional, a análise das formas de partição oferecidas pelo software FLUENT $^{\circledR} 19.2$ pertence ao pacote ANSYS $^{\circledR}$ (versão acadêmica foi utilizada), foi feita utilizando a malha com 222.295 células.

As simulações envolvendo o hidrociclone normalmente são feitas com 5 segundos com o ar desabilitado, consequentemente não há formação do air core e mais 2 com o ar habilitado para que haja a formação do air core. Sendo assim, foram feitas medições em triplicatas do tempo médio por iteração, para os tempos de simulação de $0,1,5$ e 6 segundos, esses tempos foram selecionados afim de analisar o tempo médio por iteração na inicialização, sendo representado pelo tempo de simulação de $0 \mathrm{~s}$, após a estabilização do tempo médio por iteração, sendo representação pelo tempo de simulação de $1 \mathrm{~s}$, analisar do tempo médio por iteração após a inserção de uma unidade para o valor do backflow, sendo representado pelo tempo de simulação de $5 \mathrm{~s}$ e por último o tempo médio por iteração após a estabilização do processo iterativo após a alteração do backflow, sendo este representado pelo tempo de simulação de 6 s, vale ressaltar que o desvio padrão obtido representa as oscilações da capacidade de processamento do computador utilizado. No caso específico do trabalho apresentado o computador possui 8 núcleos, consequentemente a malha foi dividida em 8 subdomínios. 


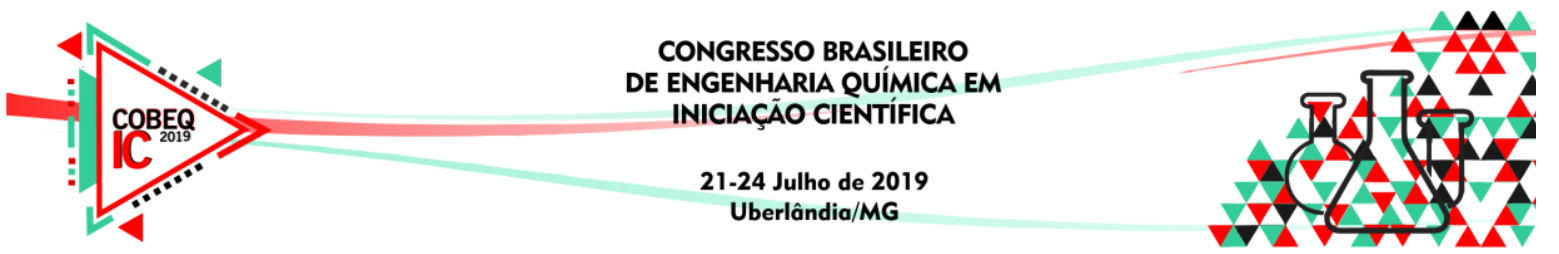

Essa análise foi feita utilizado um número de passos de tempo (Number of Time Steps) de 100, sendo que, esse parâmetro representa o número de iterações. Para o instante de zero segundos o passo de tempo utilizado foi de $10^{-6}$ e nos demais tempos foi utilizado $10^{-5}$, pois quanto maior o passo de tempo, maior serão os resíduos associados a simulação, podendo assim, fazer com que o erro ultrapasse o critério de convergência adotado que é $10^{-4}$, vale salientar que com o que conforme o passo de tempo é aumentado o tempo médio por iteração também aumenta.

\section{RESULTADOS E DISCUSSÕES}

As Tabelas 1 e 2, contém os valores de tempo médio por iteração com seu desvio padrão, para o tempo de simulação de $0 \mathrm{~s}$ e para o tempo de simulação de $1 \mathrm{~s}$, respectivamente, logo para os tempos de simulação anteriores a alteração do valor do backflow.

Tabela 1 - Tempo médio por iteração para o tempo de simulação de 0 segundos

\begin{tabular}{cc}
\hline \multicolumn{2}{c}{ Tempo de simulação de $\mathbf{0 , 0}$ segundos } \\
\hline Modelos & Tempo/iteração (s) \\
\hline Cartesian Y-Coord. & $0,376 \pm 0,001$ \\
Metis & $0,376 \pm 0,004$ \\
Spherical Rho-Coord. & $0,377 \pm 0,002$ \\
Cartesian RX-Coord. & $0,377 \pm 0,003$ \\
Cartesian Strip & $0,378 \pm 0,001$ \\
Principal Strip & $0,378 \pm 0,003$ \\
Principal Z-Coord. & $0,379 \pm 0,001$ \\
Cartesian R-Axes & $0,379 \pm 0,005$ \\
Cartesian RZ-Coord. & $0,379 \pm 0,007$ \\
Cartesian Axes & $0,380 \pm 0,004$ \\
Spherical Axes & $0,380 \pm 0,002$ \\
Principal X-Coord. & $0,381 \pm 0,003$ \\
Cylindrical Axes & $0,382 \pm 0,005$ \\
Cylindrical $R$-Coord. & $0,383 \pm 0,004$ \\
Principal Axes & $0,395 \pm 0,002$ \\
Cylindrical Theta-Coord. & $0,438 \pm 0,004$ \\
Spherical Theta-Coord. & $0,440 \pm 0,002$ \\
Cylindrical Z-Coord. & $0,441 \pm 0,005$ \\
Principal Y-Coord. & $0,441 \pm 0,001$ \\
Cartesian Z-Coord. & $0,444 \pm 0,003$ \\
Cartesian X-Coord. & $0,448 \pm 0,003$ \\
Spherical Phi-Coord. & $0,453 \pm 0,004$ \\
Cartesian RY-Coord. & $0,485 \pm 0,006$ \\
\hline
\end{tabular}

Tabela 2 - Tempo médio por iteração para o tempo de simulação de 1 segundos

\begin{tabular}{cc}
\hline \multicolumn{2}{c}{ Tempo de simulação de 1,0 segundos } \\
\hline Modelos & Tempo/iteração(s) \\
\hline Spherical Axes & $0,407 \pm 0,004$ \\
Principal Z-Coord. & $0,408 \pm 0,002$ \\
Spherical Rho-Coord. & $0,409 \pm 0,004$ \\
Metis & $0,411 \pm 0,005$ \\
Cartesian Strip & $0,411 \pm 0,007$ \\
Principal Strip & $0,412 \pm 0,006$ \\
Principal X-Coord. & $0,413 \pm 0,009$ \\
Cartesian Axes & $0,413 \pm 0,007$ \\
Cartesian RZ-Coord. & $0,414 \pm 0,006$ \\
Cartesian $R X$-Coord. & $0,416 \pm 0,003$ \\
Cylindrical Axes & $0,416 \pm 0,007$ \\
Cylindrical $R$-Coord. & $0,416 \pm 0,005$ \\
Cartesian Y-Coord. & $0,420 \pm 0,006$ \\
Cartesian R-Axes & $0,420 \pm 0,005$ \\
Principal Axes & $0,427 \pm 0,001$ \\
Cylindrical Theta-Coord. & $0,461 \pm 0,002$ \\
Spherical Theta-Coord. & $0,465 \pm 0,006$ \\
Spherical Phi-Coord. & $0,472 \pm 0,005$ \\
Principal Y-Coord. & $0,480 \pm 0,007$ \\
Cartesian X-Coord. & $0,482 \pm 0,003$ \\
Caretsian Z-Coord. & $0,484 \pm 0,004$ \\
Cylindrical Z-Coord. & $0,489 \pm 0,006$ \\
Cartesian RY-Coord. & $0,528 \pm 0,001$ \\
\hline
\end{tabular}




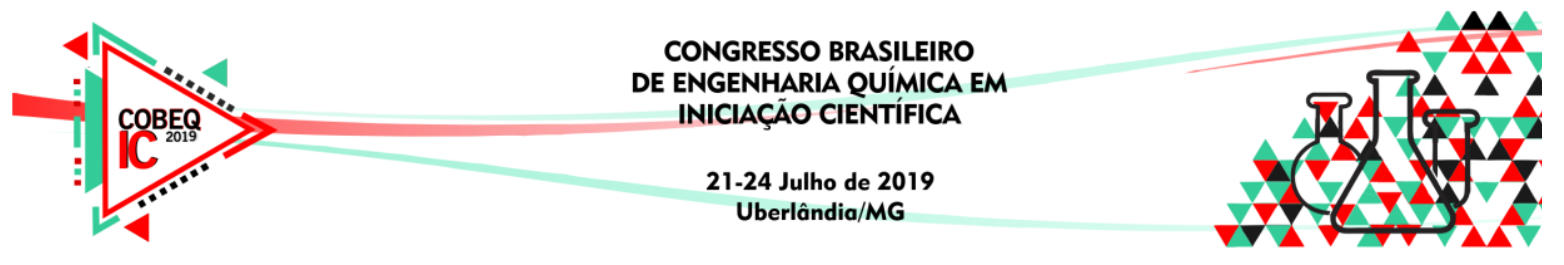

As Tabelas 3 e 4, contém os valores do tempo médio por iteração com seu desvio padrão, para o tempo de simulação de $5 \mathrm{~s}$ e para o tempo de simulação de $6 \mathrm{~s}$, respectivamente, logo posterior a alteração do valor do backflow. Vale salientar métodos estão dispostos em ordem crescente de tempo médio por iteração.

Tabela 3 - Tempo médio por iteração para o tempo de simulação de 5 segundos

\begin{tabular}{cc}
\hline \multicolumn{2}{c}{ Iteração 5,0 segundos } \\
\hline Modelos & Tempo/iteração(s) \\
\hline Spherical Rho-Coord. & $0,403 \pm 0,001$ \\
Spherical Axes & $0,408 \pm 0,006$ \\
Principal Z-Coord. & $0,409 \pm 0,004$ \\
Cartesian RZ-Coord. & $0,409 \pm 0,001$ \\
Principal Strip & $0,410 \pm 0,005$ \\
Cartesian Axes & $0,411 \pm 0,002$ \\
Cartesian R-Axes & $0,411 \pm 0,004$ \\
Cartesian Y-Coord. & $0,411 \pm 0,003$ \\
Cartesian Strip & $0,412 \pm 0,004$ \\
Cartesian RX-Coord. & $0,412 \pm 0,004$ \\
Cylindrical Axes & $0,413 \pm 0,005$ \\
Cylindrical R-Coord. & $0,413 \pm 0,008$ \\
Metis & $0,414 \pm 0,002$ \\
Principal X-Coord. & $0,415 \pm 0,006$ \\
Principal Axes & $0,425 \pm 0,003$ \\
Spherical Theta-Coord. & $0,464 \pm 0,009$ \\
Spherical Phi-Coord. & $0,464 \pm 0,003$ \\
Cylindrical Theta-Coord. & $0,469 \pm 0,007$ \\
Cartesian X-Coord. & $0,483 \pm 0,003$ \\
Principal Y-Coord. & $0,484 \pm 0,003$ \\
Cylindrical Z-Coord. & $0,484 \pm 0,004$ \\
Caretsian Z-Coord. & $0,484 \pm 0,004$ \\
Cartesian RY-Coord. & $0,528 \pm 0,005$ \\
\hline
\end{tabular}

Tabela 4 - Tempo médio por iteração para o tempo de simulação de 6 segundos

\begin{tabular}{cc}
\hline \multicolumn{2}{c}{ Iteração 6,0 segundos } \\
\hline Modelos & Tempo/iteração(s) \\
\hline Cartesian Axes & $0,406 \pm 0,002$ \\
Cartesian Strip & $0,407 \pm 0,004$ \\
Spherical Rho-Coord. & $0,407 \pm 0,003$ \\
Principal Strip & $0,408 \pm 0,008$ \\
Principal Z-Coord. & $0,411 \pm 0,004$ \\
Cartesian R-Axes & $0,411 \pm 0,002$ \\
Cartesian RX-Coord. & $0,413 \pm 0,005$ \\
Cartesian RZ-Coord. & $0,413 \pm 0,003$ \\
Cylindrical Axes & $0,413 \pm 0,002$ \\
Spherical Axes & $0,413 \pm 0,002$ \\
Metis & $0,414 \pm 0,003$ \\
Cartesian Y-Coord. & $0,415 \pm 0,002$ \\
Cylindrical R-Coord. & $0,415 \pm 0,003$ \\
Principal X-Coord. & $0,418 \pm 0,001$ \\
Principal Axes & $0,427 \pm 0,005$ \\
Spherical Theta-Coord. & $0,465 \pm 0,001$ \\
Cylindrical Theta-Coord. & $0,472 \pm 0,002$ \\
Spherical Phi-Coord. & $0,472 \pm 0,006$ \\
Caretsian Z-Coord. & $0,482 \pm 0,004$ \\
Cartesian X-Coord. & $0,483 \pm 0,005$ \\
Cylindrical Z-Coord. & $0,485 \pm 0,003$ \\
Principal Y-Coord. & $0,486 \pm 0,001$ \\
Cartesian RY-Coord. & $0,530 \pm 0,003$ \\
\hline
\end{tabular}

Analisando a Tabela 1, podemos observar que com o desvio padrão associado. ao tempo médio por iteração, do método Cartesian Y-Coord. ao Cylindrical $R$-Coord. temos valores que praticamente se superpõe, isso ocorre primeiramente pois todas as partições nesse intervalo fazem a divisão da malha de forma perpendicular ao eixo no qual o hidrociclone está disposto, sendo esse o eixo Y, e outro fato que pode ter afetado esse resultado e o tempo de simulação utilizado que de $0 \mathrm{~s}$, sendo assim, o interior do equipamento ainda não foi preenchido com fluido, e com isso, basicamente apenas a partição que contém a região da alimentação está fazendo os cálculos de continuidade e momento, as demais estão apenas atualizados os dados, já os demais resultados de tempo médio com exceção do Cartesian RY-Coord. possuem sua forma em comum, sendo todas essas partições da malha de forma perpendicular aos demais 


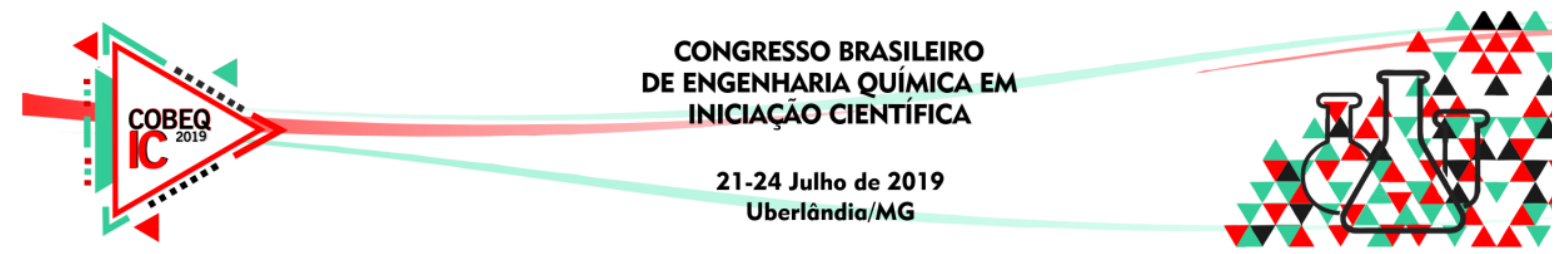

eixos, sendo esses os eixos $\mathrm{X}$ e $\mathrm{Z}$, entretanto o método Cartesian $R Y$-Coord. possui uma partição totalmente particular, sendo caracterizada por partições cilíndricas coaxiais, sobre o eixo $\mathrm{Y}$.

Analisando as Tabelas 2, 3 e 4, podemos observar o mesmo padrão, onde os menores tempos são encontrados quando a partição é formada de maneira perpendicular ao eixo no qual o hidrociclone está apoiado, e os maiores tempos quando essa divisão da malha ocorre nos demais eixos, sendo o pior método para todos os tempo o Cartesian $R Y$-Coord. que possui sua forma de partição extremamente particular, outro ponto que vale salientar e o aumento geral do tempo médio por iteração com o aumento do passo de tempo da simulação, sendo este $10^{-6}$ para o tempo de simulação de $0 \mathrm{~s}$ e $10^{-5}$ para os demais tempo de simulação analisando. A Figura 2 contém a representação das principais partições disponíveis para geometrias tridimensionais do software FLUENT $^{\circledR}$ versão 19.2.

Figura 1 - Principais tipos de particionamento e suas adaptações feitas no hidrociclone HC11.

(a)

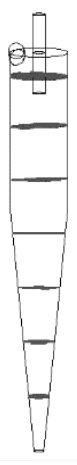

(f)

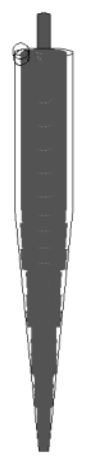

(b)

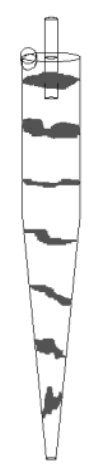

$(\mathrm{g})$

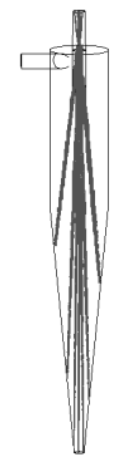

(c)

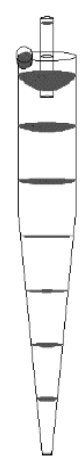

(h)

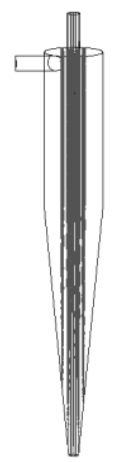

(d)

(e)

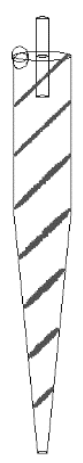

(i)

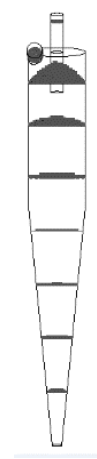

(j)
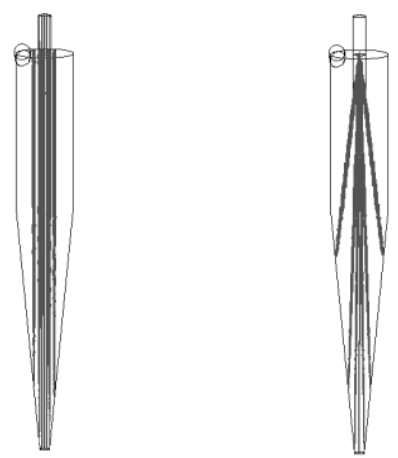

(a) Cartesian Y-Coord. /Cartesian Strip (b) Metis (c) Spherical Axes/ Spherical Rho-Coord. (d) Principal Z-Coord. / Principal Strip (e) Cartesian R-Axes / Cartesian RX-Coord. (f) Cartesian RY-Coord (g) Spherical Phi-Coord. (h) Cylindrical Z-Coord. (i) Cartesian XCoord. (j) Spherical Theta-Coord. 


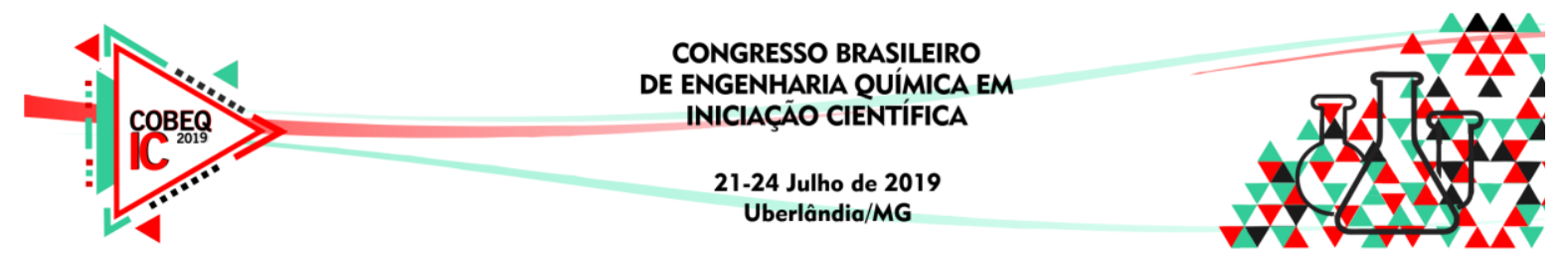

\section{CONCLUSÕES}

Portanto, podemos concluir de maneira qualitativa que as melhores partições são aquelas na qual a malha é particionada perpendicularmente ao eixo no qual o equipamento está apoiado, sendo no caso específico desse trabalho o eixo Y, já as piores partições são aquelas na qual a divisão da malha é feita perpendicularmente aos demais eixos, no caso específico do trabalho os eixos $\mathrm{X}$ e $\mathrm{Z}$, sendo essa uma análise mais geral dos resultados dos métodos de particionamento, entretanto dentro dessas situações ainda há métodos mais eficientes e menos eficientes, sendo o Spherical Axes e Spherical Rho-Coord. os mais eficientes no geral, vale ressaltar que ambos possuem a mesma partição da malha, o primeiro escolhe a coordenada esférica que minimiza a área de interface de partição, já o segundo utiliza a coordenada esférica Rho para efetuar a partição da malha como pode ser observado na Figura 1.c, já o pior método de partição é o Cartesian RY-Coord. que possui uma partição totalmente particular, formada por regiões cilíndricas coaxiais como pode ser observado na Figura 1.f.

No entanto, as diferenças do tempo médio por iteração são pequenas, analisando apenas uma iteração, mas para todo o processo pode acarretar um grande economia de custo computacional, para a simulação do hidrociclones são necessários em média 727000 iterações, sendo inicialmente o tamanho do passo de tempo igual a $10^{-6}$ e posteriormente igual $10^{-5}$, a utilização do melhor para pior método de partição para cada tempo de simulação, pode ocasionar uma economia de 24,56 horas aproximadamente, para a simulação do hidrociclone, representado aproximadamente $20 \%$ do tempo total de simulação.

\section{AGRADECIMENTOS}

Os autores deste trabalho agradecem o apoio técnico-financeiro da FAPEMIG, CNPq e UFLA destinado à execução deste projeto.

\section{REFERÊNCIAS}

ANSYS, Inc. ANSYS Fluent user's guide. Southpointe. Canonsburg, 2018.

PETRI JUNIOR.; C. R. DUARTE.; L.G. M. VIEIRA. Aplicações das técnicas de CFD para predição da distribuição de vazões volumétricas em hidrociclones dispostos em distintas baterias. Enemp, 2009.

VIEIRA, L. G. M. Otimização dos Processos de Separação em Hidrociclones Filtrantes. Tese de Doutorado apresentada à Universidade Federal de Uberlândia, 2006. 